\title{
Query Processing Using Negative and Temporal Tuples in Stream Query Engines
}

\author{
Marcin Gorawski and Aleksander Chrószcz \\ Silesian University of Technology, \\ Institute of Computer Science, \\ Akademicka 16, 44-100 Gliwice Poland \\ \{Marcin.Gorawski, Aleksander.Chroszcz\}@polsl.pl
}

\begin{abstract}
In this paper, we analyze how stream monotonicity classification can be adopted for the introduced developed model, which processes both temporal and negative events. As we show, information about stream monotonicity can be easily used to optimize individual stream operators as well as a full query plan. Comparing our stream engine with such engines as CEDR, STREAM and PIPES we demonstrate how a primary key constraint can be used in different types of the developed stream schemes. We implemented all of the above techniques in StreamAPAS.
\end{abstract}

\section{Introduction}

The definition of time is the foundation of all stream processing systems. There are unitemporal, bitemporal and tritemporal stream models. The unitemporal stream model, adopted by many existing stream systems, describes events with timestamps belonging to an application domain, whereas the bitemporal stream model supports both application time and local processing time $([1])$.

The current unitemporal systems can be classified into two groups: one representing events whose lifetimes are known a priori (e.g. [23]) and the other comprising events whose lifetimes also depend on the facts that will appear later (e.g. [4 567]). The second approach to data stream processing is more general. The lifetime of a single process is decomposed into a positive and a negative tuple that represent the beginning and the end of the lifetime, respectively. It is worth noticing that a single positive tuple does not carry the information of its exact or potential duration as it carries a temporal tuple which contains a start timestamp and an end timestamp.

Our objective is to create a unitemporal stream model which incorporates positive/negative and temporal tuples. Thanks to this it is easier to develop new operators in comparison with more general models such as [81]. Having joined temporal and positive/negative approaches, we can more easily control internal tuple collections of stream operators using both the event approach and the temporal approach. As a result, we can monitor not only the current operator state but also predict its duration.

Another challenge in designing a continuous query system is to minimize the amount of storage and computation. In order to show the advantages of our approach, we compare the join, aggregate, minus and distinct operators defined in different stream processing systems. 
Additionally, we also adapt the monotonicity classification [9] to our model in order to optimize implementations of stream operators. This information is also helpful during the optimization of the full query plan.

\section{Concept of Data Stream Processing}

\subsection{Tuple Lifetime}

In contrast to such stream processor architectures as [2/7/3 5/8 10] our stream definition incorporates positive temporal tuples and negative tuples. Let $\mathrm{T}$ be a discrete time domain. Let $\mathrm{I}:=\left\{\left[t_{\mathrm{s}}, t_{\mathrm{e}}\right) \mid t_{\mathrm{s}}, t_{\mathrm{e}} \in \mathrm{T} \wedge t_{\mathrm{s}} \leq t_{\mathrm{e}}\right\}$ be the set of time intervals.

Definition 1. Stream: A triple $S=\left(\mathrm{M}, \leq_{t_{\mathrm{s}}, t_{\mathrm{e}}}, p k\right)$ is a stream, if:

$\mathrm{M} \quad-$ is an infinite sequence of tuples (type, $e,\left[t_{\mathrm{s}}, t_{\mathrm{e}}\right)$ ),

where:

type-tuple type,

$e$-attributes,

$\left[t_{\mathrm{s}}, t_{\mathrm{e}}\right) \in \mathrm{I}-$ start timestamp and end timestamp, respectively.

$\leq_{t_{\mathrm{s}}, t_{\mathrm{e}}}$ - is the lexicographical order relation over $\mathrm{M}$

(primarily sorting by $t_{\mathrm{s}}$ and secondarily by $t_{\mathrm{e}}$ ).

pk - attributes of primary key.

The tuples are of two types: positive temporal and negative. The end timestamp of a positive temporal tuple defines the upper boundary of the tuple lifetime. When lifetimes of tuples are known at the time of their generation, the start and end timestamps define the exact lifetimes. When we do not know the lifetimes of tuples at the time of their generation, the end timestamps determine the upper boundary of the tuple lifetimes. Negative tuples expire positive tuples according to the primary key value. In comparison with positive temporal tuples, negative tuples have zero lifetime periods and are represented in figures by points.

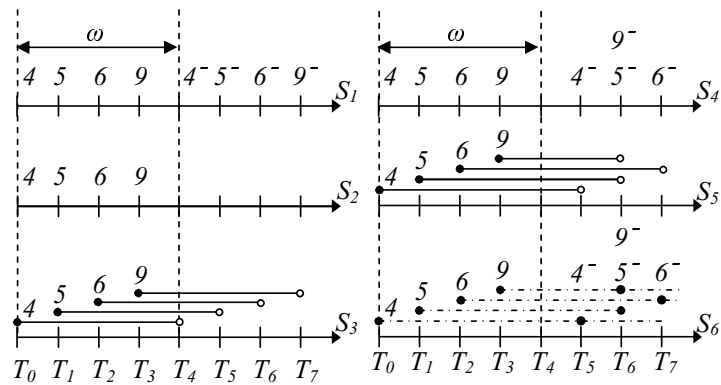

Fig. 1. Different stream models

In order to show the flexibility of the defined stream model we compare it with the existing models. 
Let us assume that we are monitoring some real phenomenon, which consists of elementary processes. They are described by attributes and timestamps. Streams in Fig. 11 illustrate the observation of the same elementary processes but they are represented by a different stream model. Stream $S_{1}$ uses two tuples so as to define the lifetime of an event. Positive tuples signal the beginnings of events and negative ones show the ends. Knowing the application time and the lifetime period, we can define a time window [7] which translates the input stream into the collection of valid tuples. For instance, when the lifetimes of the above processes are constant and equal $\omega$, we can only transmit positive tuples as it is shown on $S_{2}$. When we know the exact lifetimes of tuples at the time of their creation, we can use temporal tuples [2] which contain start and end timestamps, as it is shown on $S_{3}$. The main advantage of temporal tuples is that they reduce the amount of transmitted data twice, which is shown on $S_{3}$. On the other hand, it becomes complicated to use temporal tuples [2] when their lifetimes are not known at the time of their creation. In such a case, time is divided into smaller periods. When a period elapses, there are produced temporal tuples which prolong the lifetimes of previously sent tuples that are still valid. Nevertheless, this solution generates a lot of additional data.

The observation of another real phenomenon is illustrated by streams $S_{4}, S_{5}$ and $S_{6}$. Let us assume that we don't know the lifetimes of events at the time of their creations. As a result we cannot represent them directly as it is shown on stream $S_{5}$. The integration of the temporal stream model and the model of streams with positive and negative tuples allows as to solve this problem. An example of this model is stream $S_{6}$. In this scenario, if we know that a tuple will have been expired by a given time, we assign this value to the end timestamp. Otherwise we assign infinity. In order to distinguish between positive temporal tuples and temporal ones, the broken lines represent the lifetimes of positive temporal tuples.

\subsection{Stream Monotonicity}

Depending on the source type, streams transmit either only positive temporal tuples or both positive temporal tuples and negative ones. The content of streams can be classified according to their monotonicity. As we show further, this property enables the stream database to optimize the implementations of operators by using more efficient tuple collections. In the stream processing system, we borrow the stream monotonicity classification from [9]. Let $Q$ be a query and $\tau$ a point in time. Assume that at $\tau$, all tuples with lower or equal timestamps have already been processed. The multi-set of input tuples at time $\tau$ is denoted as $S(\tau)$, whereas all the tuples from time 0 to time $\tau$ are denoted as $S(0, \tau)$. Furthermore, let $P_{S}(\tau)$ be the result multi-set produced at time $\tau$ and let $E_{S}(\tau)$ be the multi-set of expired tuples at time $\tau$. The equation below defines the function of the result set update.

$$
\forall \tau Q(\tau+1)=Q(\tau) \cup P_{S}(\tau+1)-E_{S}(\tau+1) .
$$

The types of stream monotonicity are defined indirectly. Using the above symbols we define operators which generate streams of a given monotonicity:

1. The monotonic operator is an operator that produces result tuples that never expire. Formally the property is described as follows: $\forall \tau \forall S: E_{S}(\tau)=0$. 
2. The weakest non-monotonic operator is an operator that produces result tuples whose lifetime is known and constant. Thanks to this the order in which those tuples appear at the operator input corresponds to the order of their expiration. This can be formalized as: $\forall \tau \forall S \exists c \in \mathrm{N}: E_{S}(\tau)=P(\tau-c)$.

3. The weak non-monotonic operator is an operator whose result tuples have different lifetimes but they are still known at the time of their generation. Let us note that the order of tuple insertion and the order of their expiration are different. Formally represented, it looks like: $\forall \tau \forall S \forall S^{\prime}: S(0, \tau)=S^{\prime}(0, \tau)$, it is true that $\forall t \in P_{S}(0, \tau) \exists e: t \in E_{S}(e) \wedge t \in E_{S}^{\prime}(e)$.

4. The strict non-monotonic operator is an operator whose expiration of tuples depends on the input tuples that will arrive in the future. The lifetimes of tuples are not known at the time of their generation. This can be formalized as follows: $\exists \tau \exists S \exists S^{\prime}: S(0, \tau)=S^{\prime}(0, \tau)$ and $\exists e \exists t \in P_{S}(0, \tau)$ then $t \in E_{S}(e) \wedge t \notin E_{S}^{\prime}(e)$.

The monotonicity of type one says that tuples of a given stream never expire. This means that streams of this type transmit only positive temporal tuples with infinity assigned to end timestamps. The monotonicity of type two is illustrated by $S_{3}$ in Fig. 1 Stream $S_{5}$ exemplifies a stream with monotonicity of type three. The last type of monotonicity is illustrated by $S_{6}$.

It is worth noticing that the higher the number of stream monotonicity, the more complicated the architecture of the tuple collection which is linked to a given stream. Collections connected with streams of monotonicity: 1, 2 and 3 process no negative tuples. Those collections check only end timestamps so as to find expired tuples. If a stream is of the weakest non-monotonic type, the order of tuple expiration and their arrival order are the same. As a result, if a simple list data structure is used, then potentially expired tuples exist only at its beginning. The stream monotonicity of type three has two potential implementations. The expired tuples can be identified by testing all the elements of a collection or we can add auxiliary indexes which store tuples in the end timestamp order.

According to [7], positive tuples and corresponding negative ones have the same values of attributes except for the flag which distinguishes types of tuples. In order to speed up finding positive temporal tuples which correspond to given negative tuples, we have introduced the primary key (PK) into stream schemes. If a stream of strict non-monotonic type has a defined PK, then we can create an index which simplifies processing negative tuples. In a traditional DBMS, the PK not only identifies records in relations but also excludes duplicates from relations. Similarly, if an operator is attached to a strict non-monotonic stream, it has to filter out duplicates and does not process them.

\section{Stream Operators}

It is worth noticing that the chosen stream model determines the lowest latency of result tuples which can be theoretically achieved. The stream model with temporal tuples assumes that the lifetime of generated tuples can't be changed (to be more precise shortened). In consequence, some state-full operators (e.g. the aggregation operator) produces only tuples which elapse by $t$ at time $t$. In summary, the effect of processing 
temporal tuples $\left[t_{s}, t_{e}\right)$ will be shown at an output stream after processing another tuple that has a grater $t_{s}$ value. In contrast to that, operators defined in a stream model with positive/negative tuples always generate the result tuples which correspond to the most recent state of an operator. Despite the fact that our stream model uses positive temporal tuples, it doesn't have the above drawback.

\subsection{Join Operator}

In Fig.2 we compare join operators for: a) the model with positive and negative tuples; b) the temporal model; and c) the mixed model. Streams $I n_{1}$ and $I n_{2}$ are the input streams and Out is the result of a join operator in Fig. 26). Stream $I n_{1}$ transmits only positive temporal tuples and $\mathrm{In}_{2}$ transmits both types of tuples. Let us notice that the result streams Out in Fig. 2b) and Fig. 2k) transmit the same tuples but they have a different interpretation. The result tuples in Fig. 2b) define their lifetimes precisely, whereas the result tuples in Fig. 2k) define the upper boundaries of their lifetimes. In contrast to the stream model with positive and negative tuples, there is no negative result tuples in Out. This situation occurs because the negative tuples arrive in $I n_{2}$ later than the upper lifetime boundary of result tuples in Out.

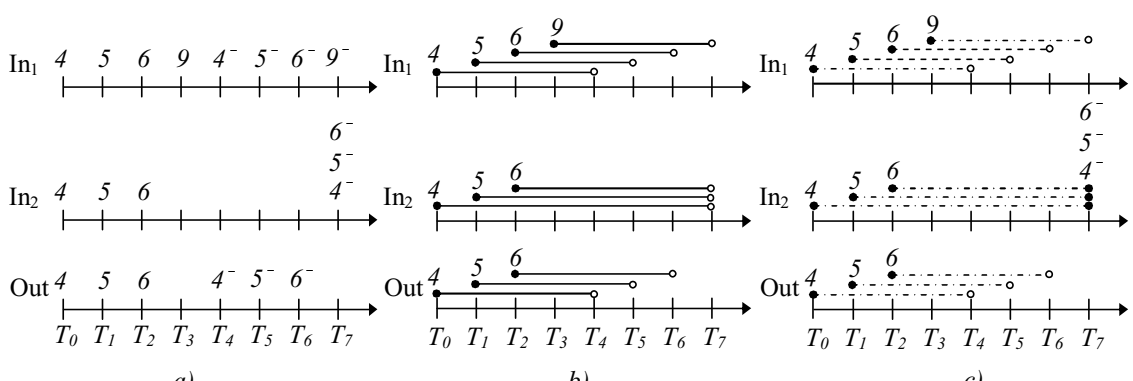

Fig. 2. Join operators for different stream models

\subsection{Aggregate Operator}

In order to emphasize differences between implementations of aggregate operators, we analyze examples of calculating sum aggregates. Although the aggregate operator can have a defined grouping function on tuple attributes, in order to make figures easier to follow, we analyze aggregate operators without them.

1. Figure 34) shows an operator designed to process temporal tuples [2] only. After a new tuple $t$ enters the operator, the output stream is fed with result tuples whose end timestamp is lower or equal to the start timestamp of $t$. This operator puts forward only aggregates whose lifetimes are fully defined. As a result the operator propagates the current values of aggregates when it receives another tuple.

2. The operator designed to process positive/negative tuples [7] is shown in Fig. 3b). In order to make this figure easier to follow, we have put the corresponding positive 


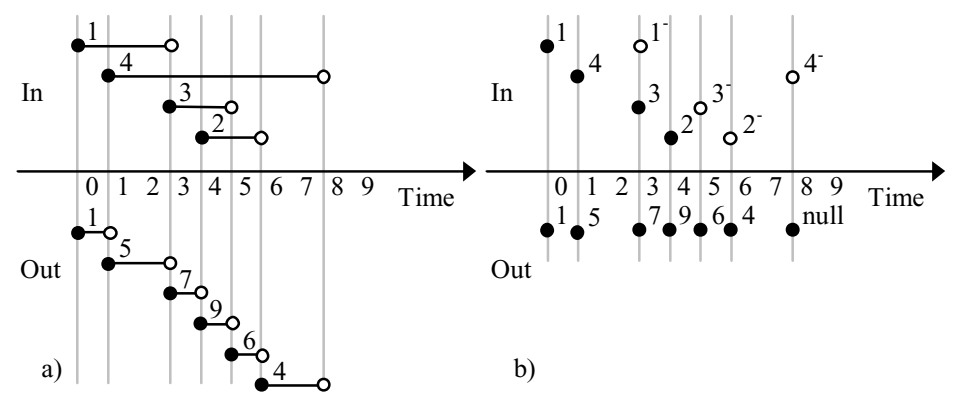

Fig. 3. Aggregate operators for different stream models: a) temporal tuples, b) positive/negeative tuples

and negative tuples on the same level. This operator recalculates the aggregate value and pushes it to the output right after receiving le. Additionally, if we assume that the latest output value for a group overrides the previous value for the same group then this operator does not produce negative tuples. Moreover, after processing the last input tuple for a given group, the operator produces null tuple for this group [7].

3. The aggregate operator that processes streams of type 3) (Fig. 4 ) produces a tuple with the current aggregate value immediately after receiving a new tuple, similarly to the above operator. Aggregates are represented by positive temporal tuples. The lifetime of tuple $t$ that represents the aggregate belonging to group $g$ tells us when the aggregate value finally expires according to tuples received by $g$ before $t$ was produced. The output of this operator is always of strict non-monotonic type.

Now we summarize the aggregate operator for the above stream models:

1. The aggregate operator that processes temporal tuples is always delayed by one tuple. In consequence, we observe the old aggregate value until a new tuple arrives. In order to shorten the delay, the authors [2] suggest dividing the lifetime of an entering tuple into smaller periods. As a result the aggregate operator more frequently refreshes the output stream. However, it generates more tuples which have to be processed.

2. The aggregate operator that processes positive/negative tuples is free of the above imperfection. Figure 3 b) shows a solution which generates only positive tuples. Let us assume that the aggregate operator has defined grouping attributes. In this case, when new result tuple $t_{n e w}$ is produced, it overrides the previous aggregate value of the same group as $t_{n e w}$. In consequence, successive operators attached to this aggregate operator have to know the definition of the grouping function so as to detect tuples expiration correctly. As a result, attaching subsequent operators to such an aggregate operator becomes complicated. If we want to avoid this drawback, the operator has to produce both positive and negative tuples.

3. Considering our stream model, lifetimes of tuples are determined by either end timestamps or negative ones. We do not make use of the assumption that a new tuple overwrites the previous one. Consequently, linking aggregate operators in a query plan is easy. Moreover, the result aggregates are propagated to the output when a new tuple arrives, like in the previous operator description. 


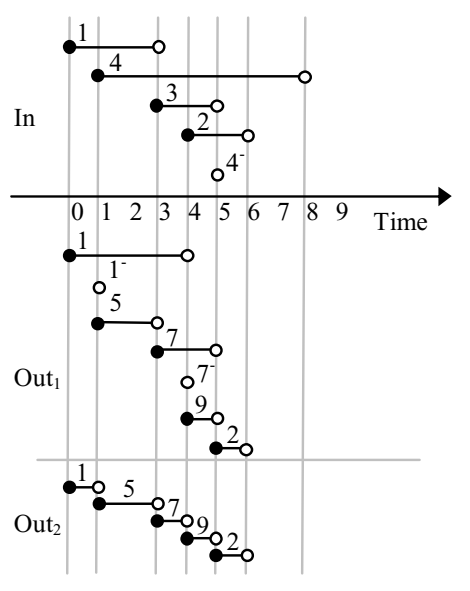

Fig. 4. Aggregate operator which process positive temporal and negative tuples

Now let us compare the aggregate operators with the defined grouping attributes. The input stream is divided into $N_{g r}$ groups.

The aggregate operator for model 1) generates result tuples for all $N_{g r}$ groups after processing a new input tuple because all aggregates of groups have to be propagated to the output. The explanation of it is as follows: when a new aggregate is sent to the output it has to have a defined exact lifetime. In consequence it is generated when another tuple arrives at the input. Having $N_{g r}$ groups of aggregates, the next input tuple triggers the calculation for each group.

The aggregate operator for model 2) updates the aggregate value of the group affected by an input tuple. The number of result tuples depends on the operator configuration. It can generate either positive and negative tuples or only positive tuples like in Fig. 3 b). Even if we consider the configuration which generates positive and negative tuples, it generates less tuples than the operator defined for mode 1).

The aggregate operator in model 3) updates the aggregate value of the group affected by an input tuple, similarly to the operator defined on model 2). Positive temporal tuples determine lifetime boundaries. Thanks to this the operator does not produce as many tuples as the operator in model 1).

\subsection{Stream Compression}

When DSMS is loaded, more tuples are stored in stream buffers. In consequence, a stream operator processes a packet of tuples in one round of a scheduler assignment [11]. Let us assume that the aggregate operator buffers the result tuples while processing this packet. Having processed the last tuple of the packet, the buffer consists of positive temporal tuples and negative ones. Now we can check if each negative tuple $t_{n}$ corresponds to a positive temporal tuple $t_{p}$ which also appears in this buffer. If the above conditions are met, we can reduce those tuples as follows: a positive temporal tuple $t_{p}$ has updated $t_{p} . t_{e}=t_{n} . t_{s}$ and a negative tuple $t_{n}$ is removed. Finally, the buffer is compressed and subsequently transmitted to the output stream. 


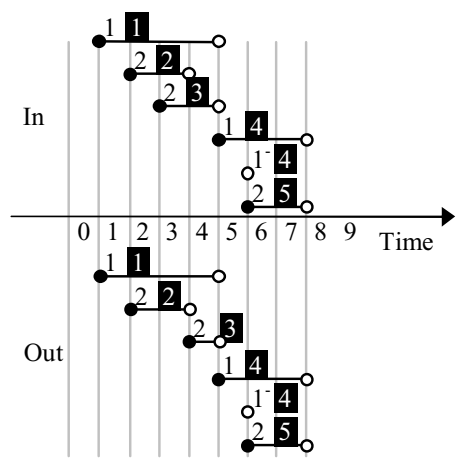

Fig. 5. A sample output of the distinct operator attached to a strict non-monotonic stream

\subsection{Distinct Operator}

The distinct operator uses an internal collection, which contains input tuples as long as they are valid. The input tuple has attributes $D S T_{A t t r}$ which represent distinct values. When a positive temporal tuple $t_{1}$ arrives, the internal operator collection is checked for the existence of $D S T_{A t t r}$ values of $t_{1}$. If not, $t_{1}$ is propagated to the output and added to the collection. Otherwise it is only added to the collection. When $t_{1}$ is terminated by either the lifetime limit or the corresponding negative tuple, the distinct operator checks if there is a valid tuple with the same $D S T_{A t t r}$ values as $t_{1}$. If there is such a tuple $t_{\text {next }}$, the operator produces a new result which has attribute values from $t_{\text {next }}$ and the lifetime: $s t a r t=t_{1}$.end, end $=t_{n e x t}$.end. Let us consider the monotonicity of the result stream. If the operator is fed with a strict non-monotonic stream, then the result stream is also of that type. Otherwise it is of the weak non-monotonic type. A sample output of the distinct operator is shown in Fig. 5. The numbers printed in black represent $D S T_{A t t r}$, and the numbers in white are PK. Let us consider a scenario in which negative tuple $2^{-}$arrives at $t=4:\langle 2,[4,4)\rangle^{-}$. Without knowing the PK value, we cannot chose the right tuple to remove because both tuples $<2,[2,4)>$ and $<2,[3,5)>$ have the same attribute values. On the other hand, they have different lifetimes. Depending on which tuple was removed, a different result tuple will be produced. In contrast to this operator, the distinct operator defined for streams with positive and negative tuples [7] does not have to use the PK. All positive tuples represent an infinite lifetime so that if there exist duplicates, the operator removes just one of them.

The algorithm below gives the pseudo code of the distinct operator which is attached to the strict non-monotonic stream. This algorithm uses the following collections:

- Hash table, $H P K$, which stores input tuples sorted by PK.

- Collection, $H D S T$, which contains buckets of tuples sorted by $D S T_{A t t r}$.

- Collection, LExp, which stores input tuples sorted by start and end timestamp.

- Collection, $D L$, which stores tuples that generate distinct tuples.

Let us notice that the collection $H P K$ can be removed when the operator is attached to the streams of other monotonicity types. 
Operator distinct; main procedure.

Input: $\mathrm{t}$ - an incoming tuple

1 RemoveExpiredTuples $(t)$

2 If $t$ is a positive temporal tuple

3 If $t$ is not found in $H P K$

$4 \quad$ Add $t$ to $H P K$

$5 \quad$ Add $t$ to LExp

6 If HDST doesn't contain bucket for $t$

$7 \quad$ Create bucket $b$; add $b$ to HDST

$8 \quad$ Add $t$ to $b$

$9 \quad$ Add $t$ to $D L$

10 Produce positive temporal tuple to Output

11 Else

12 Add $t$ to that bucket

13 If $t$ is a negative tuple

14 If $t$ is found in $H P K$

15 Remove $t$ from $H P K$

16 Remove $t$ from LExp

17 Get bucket $b$ containing tuple $t$

18 Remove $t$ from $b$

19 If $b$.size ()$==0$

20 Remove $t$ from $D L$; produce negative tuple to Output

21 Disconnect $b$ from $H D S T$

$22 \quad$ Else If $t \in D L$

Remove $t$ from $D L$; produce negative tuple to Output

Add the next tuple from $b$ to $D L$,

Produce new distinct tuple to Output

\subsection{Minus Operator}

The minus operator calculates $S-R$ and sends the result to stream Out. This operator uses internal collections one which contains valid tuples from $S$ and the other one contains valid tuples from $R$. Tuple $t$ at input $S(R)$ has a counterpart in the $R(S)$ collection if there exists tuple $t_{R}\left(t_{S}\right)$ whose values of attributes $A_{t t r_{R}}\left(A_{t t r}\right)$ are equal to corresponding values of attributes $A_{t t r_{S}}\left(A t t r_{R}\right.$ ) of $t$. Let us consider the following scenario. Tuple $t_{R}$ arrives at input $R$ and it has its counterpart $t_{S}$ in the $S$ collection. In consequence, the operator produces a negative tuple which terminates corresponding $t_{S}$. This scenario shows that even if the input contains only positive temporal tuples the result may contain negative tuples. Summing up, the minus operator always generates a result stream of strict non-monotonic type. Fig. 6 shows a sample execution of such an operator. 
Procedure: RemoveExpiredTuples(Tuple t) definition.

It removes expired tuples from the collection of the operator.

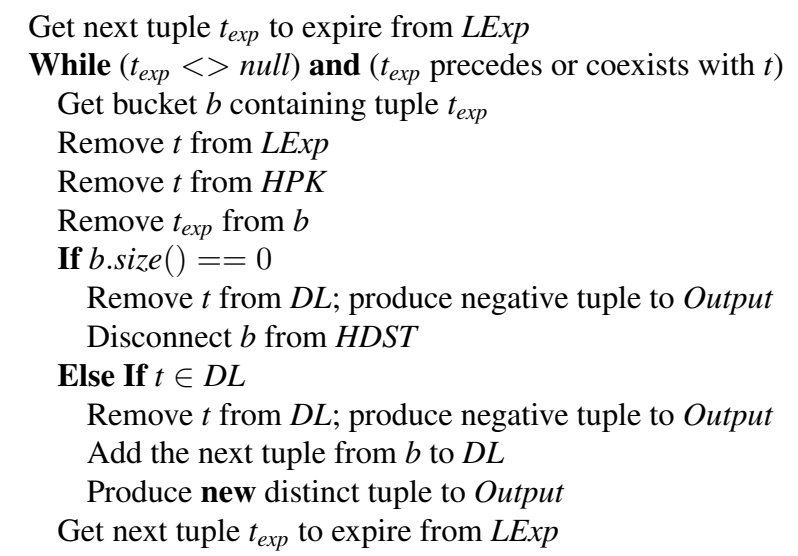

\section{Tests}

We tested the proposed stream compression algorithm in order to check how this algorithm works on the output of an aggregate operator with a defined grouping function. In this configuration, the distance between the corresponding positive temporal tuple and a negative one is higher. The number of result tuples which are generated after processing one input tuple increases as the number of groups increases. Moreover, aggregate operators produce negative tuples when the tuples that belong to the same group overlap as it is illustrated in Fig. 4. The above features make this sample query a good benchmark. Figure 7 shows the query plan, which is a chain composing of a generator, a range window, a filter operator, an aggregate operator, an map operator and a sink, respectively. We defined this test in StreamAPAS using the query statement that is shown on the right side of Fig. 7

In order to measure the compression efficiency and the latency time of the results, we present the configurations in which the aggregate operator has enabled or disabled compression. A single experiment consists of processing 1000 tuples generated by gen 1 for both configurations. Those measures were repeated for the input stream load which starts from $5000\left[\right.$ tuples/min] to $60000\left[\right.$ tuples $/$ min] produced by generator gen . $_{1}$. The generator produces a uniform load measured in [tuples/min]. Additionally, for each tuple the generator assigns a group $g \in 0 . . N$ using uniform distribution. In our experiments, value $N$ equals 100 . Figure 8 shows the changing efficiency of the compression when the system has a higher load and Fig. 9 compares the latency of result tuples. The lines named $100 \mathrm{~N}$ and $100 \mathrm{C}$ represent configurations in which compression was disabled and enabled, respectively. 


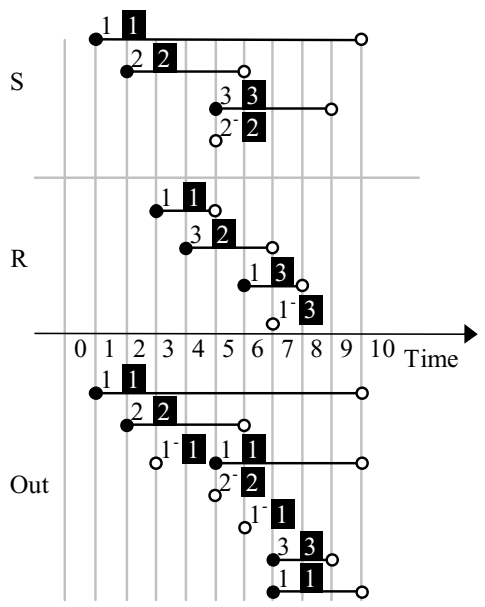

Fig. 6. The example result stream of the operator minus

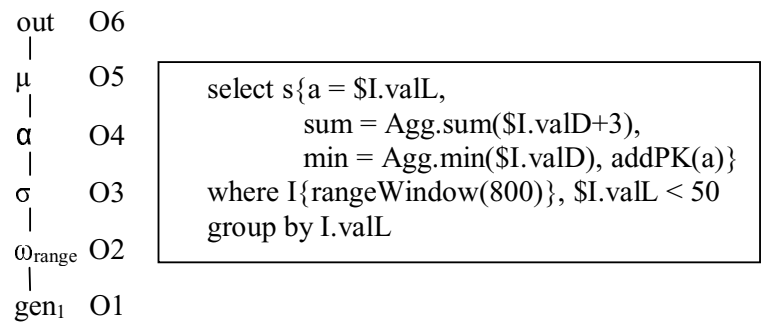

Fig. 7. The tested query

Figure 8 shows that the compression of the result stream is weaker than it could be expected. It is caused by the $N$ groups which increase the distance between the corresponding positive temporal and negative tuples. As a result, there is a low probability that a single packet contains elements that can be reduced. Figure 9 shows that the impact of compression algorithm on the result latency is huge. When the system has a low load and the compression is enabled, the latency is from $20 \%$ to $50 \%$ higher. Summing up, although the stream model that is based on positive temporal and negative tuples offers a simple stream compression algorithm, its real usability depends on the distance between the corresponding positive temporal and negative tuples. A similar compression algorithm has been introduced in the systems PIPES and CEDR. In PIPES, the context of stream compression is different. Let us assume that we have a query plan with an input stream $S$. In PIPES, the tuples of stream $S$ can be divided into a series of tuples 1 time unit in length so as to reduce the result latency of the query plan [2]. In this configuration, the stream compression efficiency is high. 


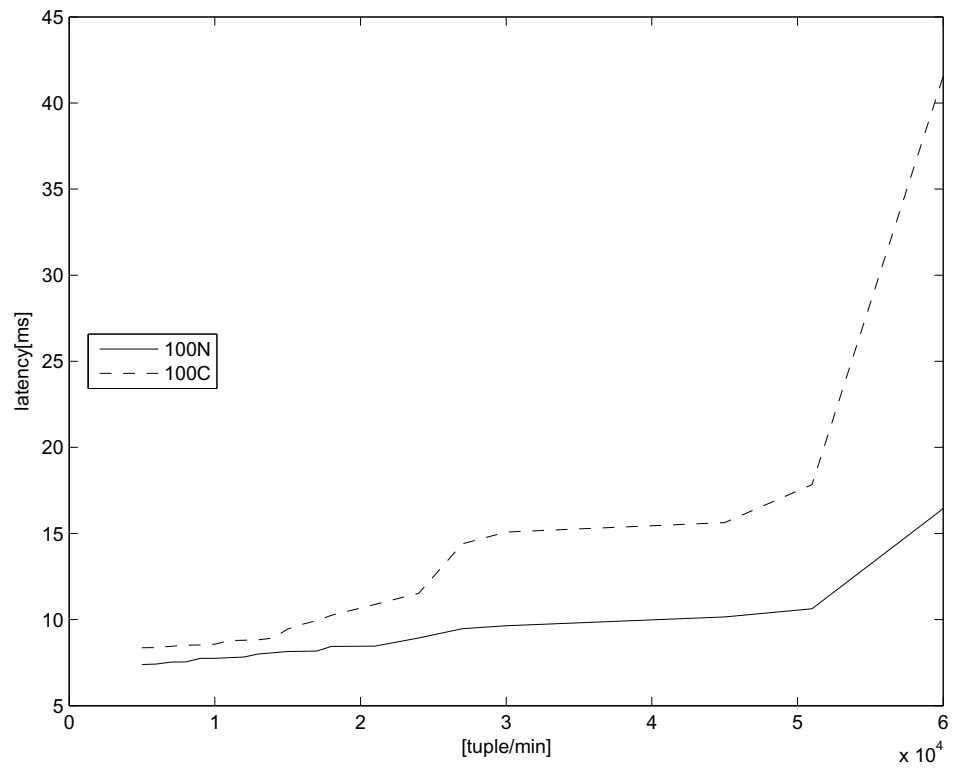

Fig. 8. Compression efficiency

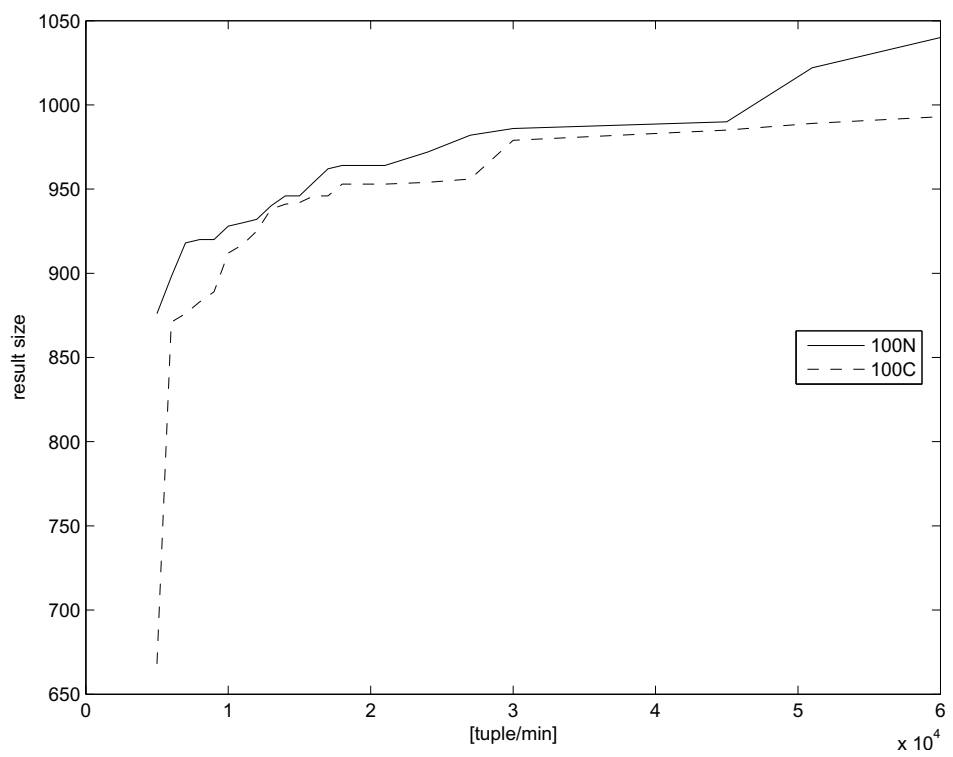

Fig. 9. The impact of compression on latency time 


\section{Conclusion}

The functionality of operators which have been introduced to stream databases comes from traditional relational databases. In StreamAPAS, we develop a stream database which adapts the basis of relational databases and temporal databases [12 13 |14] because they are also oriented towards processing ordered data. In contrast to other systems, our system processes both temporal positive tuples and the negative ones in a unitemporal stream model. As a result, this allows us to freely model an expiration time in a unitemporal stream system, which is useful in many application scenarios.

In contrast to CEDR, we have adopted stream monotonicity classification [9] and a primary key constraint. Depending on the type of monotonicity, the interpretation of a positive temporal stream can be simplified. In consequence, the stream operators do not have to maintain all collections. Additionally, the monotonicity can support the query model optimization as it was suggested in [9]. We have also introduced a primary key constraint in order to define the semantics of negative tuples. The current systems that process negative tuples assume that each tuple has only one ID attribute which identifies the corresponding tuple to be removed. This causes the problem of calculating a new ID attribute for the join operator. In our approach, we have two options. Either the user can define the function which calculates a new primary key attribute upon the attributes of the joined tuples, or the user can define the primary key composed from a few attributes.

Our work aims to research new analytical systems which we name stream data warehouses. Traditional data warehouses are designed to separate the loading process (ETL) from processing analytical queries. Stream data warehouses are to enable users to monitor the analysis in a continuous way. Therefore, we have introduced a new stream data model which joins temporal information with tuples. Thanks to this we will be able to adapt the indexes of data warehouses in the streaming model.

\section{References}

1. Barga, R.S., Goldstein, J., Ali, M.H., Hong, M.: Consistent Streaming Through Time: A Vision for Event Stream Processing. In: CIDR, pp. 363-374 (2007)

2. Krämer, J., Seeger, B.: A Temporal Foundation for Continuous Queries Over Data Streams. In: COMAD, pp. 70-82 (2005)

3. Krämer, J.: Continuous Queries Over Data Streams Semantics and Implementation. PhD thesis, Philipps-Universität Marburg (2007)

4. Babcock, B., Babu, S., Datar, M., Motwani, R., Widom, J.: Models and Issues in Data Stream Systems. In: PODS 2002: Proceedings of the 21st ACM SIGMOD-SIGACT-SIGART Symposium on Principles of Database Systems, pp. 1-16. ACM Press, New York (2002)

5. Abadi, D.J., Carney, D., Çetintemel, U., Cherniack, M., Convey, C., Lee, S., Stonebraker, M., Tatbul, N., Zdonik, S.: Aurora: a New Model and Architecture for Data Stream Management. The VLDB Journal 12(2), 120-139 (2003)

6. Balakrishnan, H., Balazinska, M., Carney, D., Cetintemel, U., Cherniack, M., Convey, C., Galvez, E., Salz, J., Stonebraker, M., Tatbul, N., Tibbetts, R., Zdonik, S.: Retrospective on Aurora. The VLDB Journal 13(4), 370-383 (2004)

7. Ghanem, T.M., Hammad, M.A., Mokbel, M.F., Aref, W.G., Elmagarmid, A.K.: Query Processing Using Negative Tuples in Stream Query Engines. Technical Report 04-040, Purdue University (2005) 
8. Tucker, P.: Punctuated Data Streams. PhD thesis, OGI School of Science \& Technology At Oregon Heath (2005)

9. Golab, L.: Sliding Window Query Processing over Data Streams. PhD thesis, University of Waterloo (2006)

10. Motwani, R., Widom, J., Arasu, A., Babcock, B., Babu, S., Datar, M., Manku, G., Olston, C., Rosenstein, J., Varma, R.: Query Processing, Resource Management, and Approximation and in a Data Stream Management System. In: Proceedings of the First Biennial Conference on Innovative Data Systems Research (CIDR 2003), Asilomar, CA, USA, pp. 245-256 (2003)

11. Babcock, B., Babu, S., Datar, M., Motwani, R.: Chain: Operator Scheduling for Memory Minimization in Data Stream Systems. In: ACM International Conference on Management of Data (SIGMOD 2003), San Diego, CA, USA, pp. 253-264 (2003)

12. Ozsoyoglu, G., Snodgrass, R.T.: Temporal and Real-time Databases: A Survey. IEEE Transaction on Knowledge and Data Engineering 7(4), 513-532 (1995)

13. Slivinskas, G., Jensen, C.S., Snodgrass, R.T.: Query Plans for Conventional and Temporal Queries Involving Duplicates and Ordering. In: Proceedings of the 16th International Conference on Data Engineering, ICDE 2000, pp. 547-558. IEEE Computer Society, Washington, DC (2000)

14. Slivinskas, G., Jensen, C.S., Snodgrass, R.T.: A Foundation for Conventional and Temporal Query Optimization Addressing Duplicates and Ordering. IEEE Transaction on Knowledge and Data Engineering 13(1), 21-49 (2001) 